\title{
A Novel Ontology Construction and Reasoning Approach Based on the Case Investigation
}

\author{
Han Zhong ${ }^{*}$, Hongzhou Zhang, Jianqian Zhang, Ziyang Yuan \\ College of Information Technology and Network Security, People's Public Security University of China, Beijing, China \\ Email address: \\ z.h0912@163.com (Han Zhong), zhanghongzhou@ppsuc.edu.cn (Hongzhou Zhang), a19680824@126.com (Jianqian Zhang), \\ effrictyuan@gmail.com (Ziyang Yuan) \\ ${ }^{*}$ Corresponding author
}

\section{To cite this article:}

Han Zhong, Hongzhou Zhang, Jianqian Zhang, Ziyang Yuan. A Novel Ontology Construction and Reasoning Approach Based on the Case Investigation. International Journal of Data Science and Analysis. Vol. 5, No. 6, 2019, pp. 148-158. doi: 10.11648/j.ijdsa.20190506.17

Received: November 8, 2019; Accepted: December 11, 2019; Published: December 31, 2019

\begin{abstract}
The big data has become a key component for intelligent systems and it is very important about data mining and cognitive reasoning in the field of criminal data analysis. Modeling of investigation knowledge is very important to realize the semantic retrieval, knowledge discovery, information push and classification for case data. Ontology modeling combined with the characteristics of the case in the investigation process, a method of ontology construction based on investigation knowledge is proposed in this paper. It builds an organization system of the investigation process at the first, which is described in stages by collecting terminology. Then the ontology of investigation knowledge is constructed. In addition, an instance is added for verification to describe the investigation process in detail. The method has a good advantage of describing the detection process quickly and integrate knowledge according to different investigation stages, formulating a standardized organization mode and providing standardized knowledge assistance in the investigation process.
\end{abstract}

Keywords: Big Data, Case Investigation, Ontology Model, Stage Construction, Clue Analysis, Semantic Reasoning

\section{Introduction}

The big data has become a key component for intelligent systems, such as intelligent vehicular networks, smart cities and it has been applied in the field of criminal data analysis. This interconnectivity together with large-scale data processing and new electronic techniques steadily brings innovation and business models of the digital space into the physical world [1-3]. It would extend tradition data technologies by incorporating knowledge from domain experts as well as the latest artificial intelligence solution, such as how to perform decision support with the case data collected by secure systems. The large amount of data is collected through secure systems to help human expert decision-making. In recent years, there are some researches about the application of big data [4]. Abdulaziz Alzubaidi explored a novel fraudulent user detection method based solely on the app usage patterns of legitimate users [5]. That presents a survey of the potential applications, architecture, modulation techniques, standardization and research challenges in visible light communication [6]. It develops an open source platform for identifying vulnerabilities in the network and facilitating wearable security research to mitigate them [7]. It is very important about data mining and cognitive reasoning, such as for smart case analysis.

In the fight against and rectification of criminal activities, case investigation is an intensive business in the field of public security and it is a main form as well [8]. Investigation knowledge is the summary and induction of intellectual achievements in public security business, which can be divided into explicit knowledge and implicit knowledge [9]. Explicit knowledge can be easily found in various papers and textbooks. Implicit knowledge exists somewhere in the theoretical expression and is contained in various public security cases. Therefore, case investigation is a kind of domain knowledge, corresponding to general knowledge. The investigation knowledge modeling of cases can satisfy the semantic retrieval, knowledge demand discovery, information push of police information knowledge and are used for the structure and classification of case data as well. The modeling of case investigation knowledge follows the process of general knowledge modeling. Case investigation 
knowledge has two aspects: case investigation and promoting investigation business. The former corresponds to the investigation theory and the latter corresponds to the investigation practice. This paper focuses on the latter. The process of case investigation needs to use meta-data to describe its information, then organizes and integrates this process or the relationship between the stages by associating knowledge. Based on the theory and method of ontology, this paper is first proposed construction model of the case investigation. According to the organization of the case investigation, the concepts, attributes and relationships are firstly defined. Then the meta-data is semantically described and the instance verification is added. Based on the above process, the content and the construction of case investigation ontology can be modeled.

\section{Background and Related Work}

With the development of knowledge engineering, the technology of ontology construction has been paid more and more attention. And it is used to construct the domain model to express the key knowledge and the relationship between domain, making final inference and prediction. At present, ontology-based methods have been applied in many fields. In some fields, standard languages of ontology modeling such as OWL have been used to describe domain content [10]. However, there is no complete ontology of constructing intention content analysis about how to judge the suspects' intentions based on known clues in the field of case investigation. And there is no effective analysis content of the clues in the environment of investigation cases, which makes it difficult to indicate the suspects' intentions and investigate the semantic content. In the process of case investigation, the analysis and research of potential clues are the key factors for effectively targeting suspects and investigating cases. It is an effective way to use the case ontology modeling for clue analysis, which must be built on the basis of modeling ontology for the case investigation. In recent years, there has been a lot of researches about ontology construction in some fields at home and abroad. The ontology construction is mostly applied in e-commerce domain [11], the domain of knowledge representation [12-14], the research of knowledge reasoning construction [15-17] and other fields [18-20]. Meanwhile, it is often used in the field of knowledge mining based on the ontology modeling [21-23] and so on. In the military field, it is common to study the ontological modeling of enemy tactical intentions [24, 25], the construction of combat operations ontology [26] and the description of complex video content with ontology framework $[27,28]$ etc. In the security field, the application of ontology construction can be traced back from the early network ontology construction of forensics analysis [29] to terrorist organization ontology $[30,31]$, criminal ontology and knowledge reasoning [32, 33] in recent years. However, semantic understanding of content needs specific background knowledge. Meanwhile, in the field of case investigation lacks unified semantic description and in the process of the case investigation also lacks research about ontology construction. Therefore, this paper applies the theory of ontology modeling to case investigation. It builds the knowledge base of domain ontology by collecting key concepts in this field and describing the relationship between concepts. With the increase of clue resources, it's urgent to find intelligent methods to understand, store, index and retrieve the clue data at the semantic layer in the process of case investigation. To facilitate the case investigation for the police, the semantic description of shared clues requires unified definition in the conceptual semantic content. Therefore, it is a certain trend to use the ontology-normalized concepts of clues analysis in the process of case investigation.

This paper focuses on applying ontology theory to build the investigation knowledge models combined with the implementation process of case investigation. This method can be applied to the actual practice of case investigation to improve the traditional pattern of clue analysis. It serves for accomplishing the analysis of potential clues and provides a foundation for predicting the trend. In this paper, cases-added ontology construction and reasoning experiments verify the proposed ontology modeling method.

\section{Foundation of Case Investigation}

\subsection{The Implementation Process Based on Ontology Theory}

The ontology of investigation knowledge is domain ontology. It is necessary to establish a domain knowledge ontology base under the experienced police in public security field. The implementation details included collecting relevant data, storing data, describing investigation knowledge in a standardized and unified language. It makes some exploration to realize the automated process of case investigation. And it also helps organizing information, formalizing domain knowledge, storing data and connecting information through semantic network. Domain experts can use the constructed ontology to evaluate, extend, adjust and update this ontology. The detail of ontology construction are shown in Figure 1.

The ontology construction firstly determines whether there is a reusable ontology. If it is a reusable ontology, it can evaluate and evolve the ontology. If it is not, it needs to collect terms, construct every stage, construct the relationship and evolve the ontology. Among them, the stage construction is the core part. It is necessary to define the scope of each stage, identify concepts, attributes and relationships, express the key content through formalization in the end. 


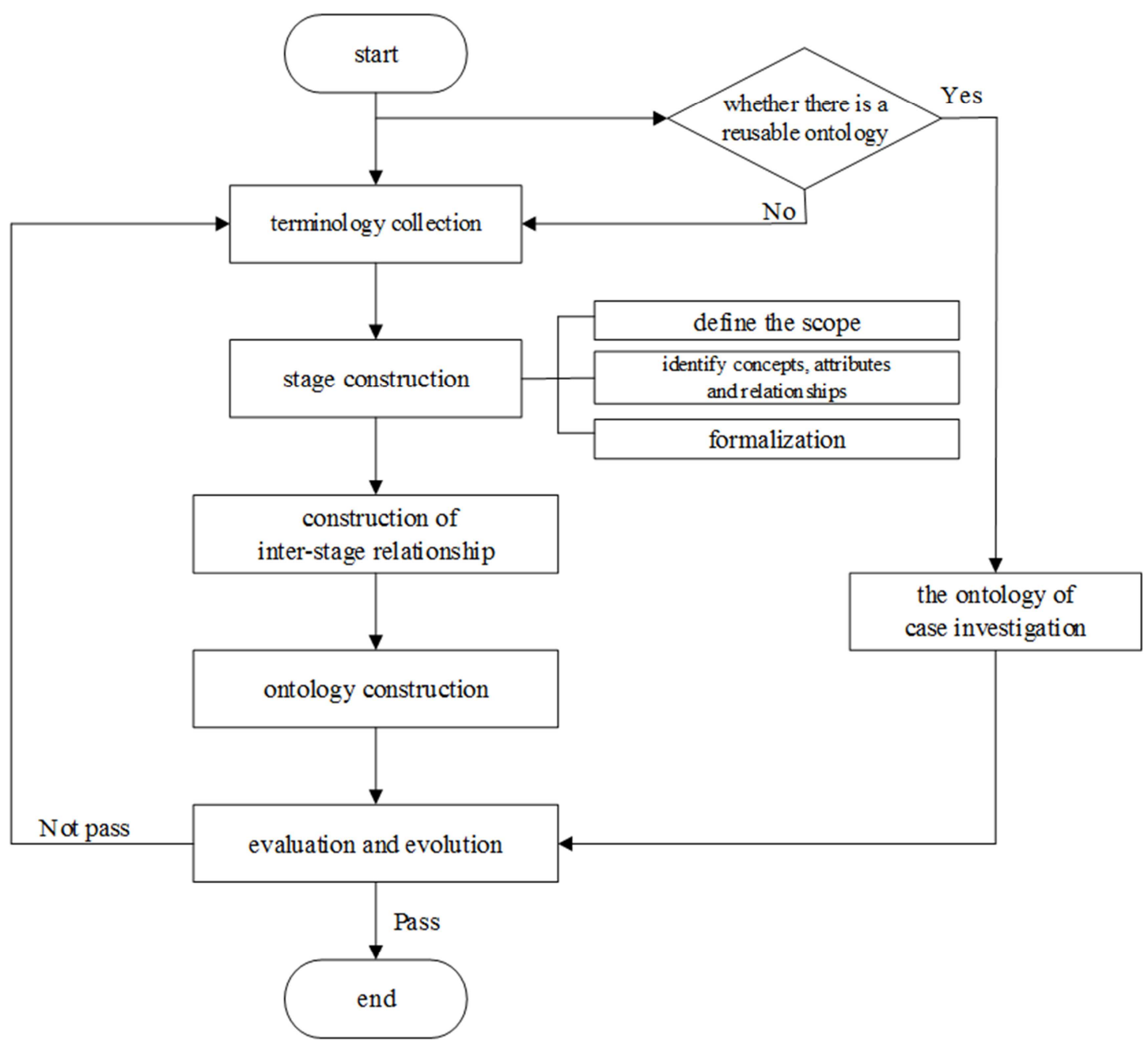

Figure 1. The process of ontology construction.

\subsection{Organization of Case Investigation}

Nowadays, the rapidly increase of information and digital organization has become an important way in the process of criminal investigation. And it is also an important basis for the current investigation or handling of cases. With the improvement of big data and intelligent level, the drawbacks of the original mode are gradually appeared. The original data of each stage can't reflect the connection between the investigation processes. Therefore, this paper constructs the case investigation knowledge organization, which can better realize the connection between investigation processes through computer language. And it also organizes and integrates useful data to realize data sharing so as to provide help for investigators. The organization of investigation process is shown in Figure 2.

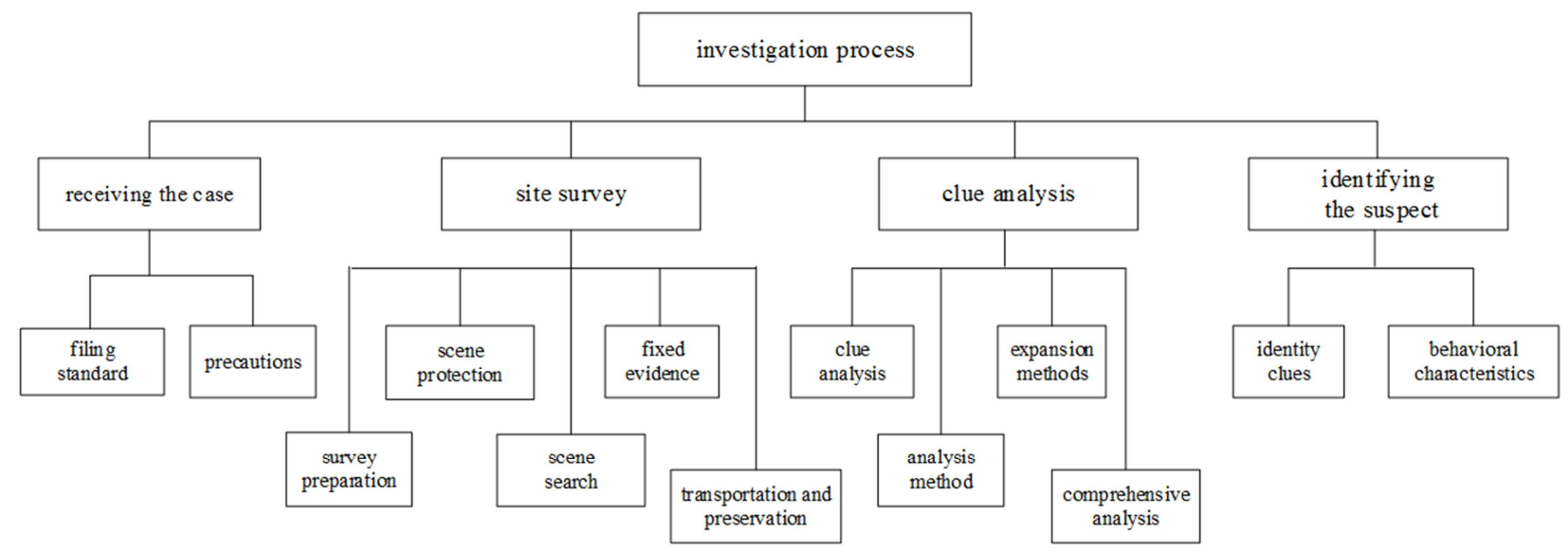

Figure 2. The organization of investigation process. 


\section{The Construction of Case Ontology Model}

\subsection{Terminology Collection}

Collecting terms is to summarize and collect some key words in the field of case investigation. Terms are the most basic part of building an ontology. Through domain analysis and expert review, we choose the method of document description to collect terms from the current system [34]. The fragment ontology of collected terms is shown in Figure 3.

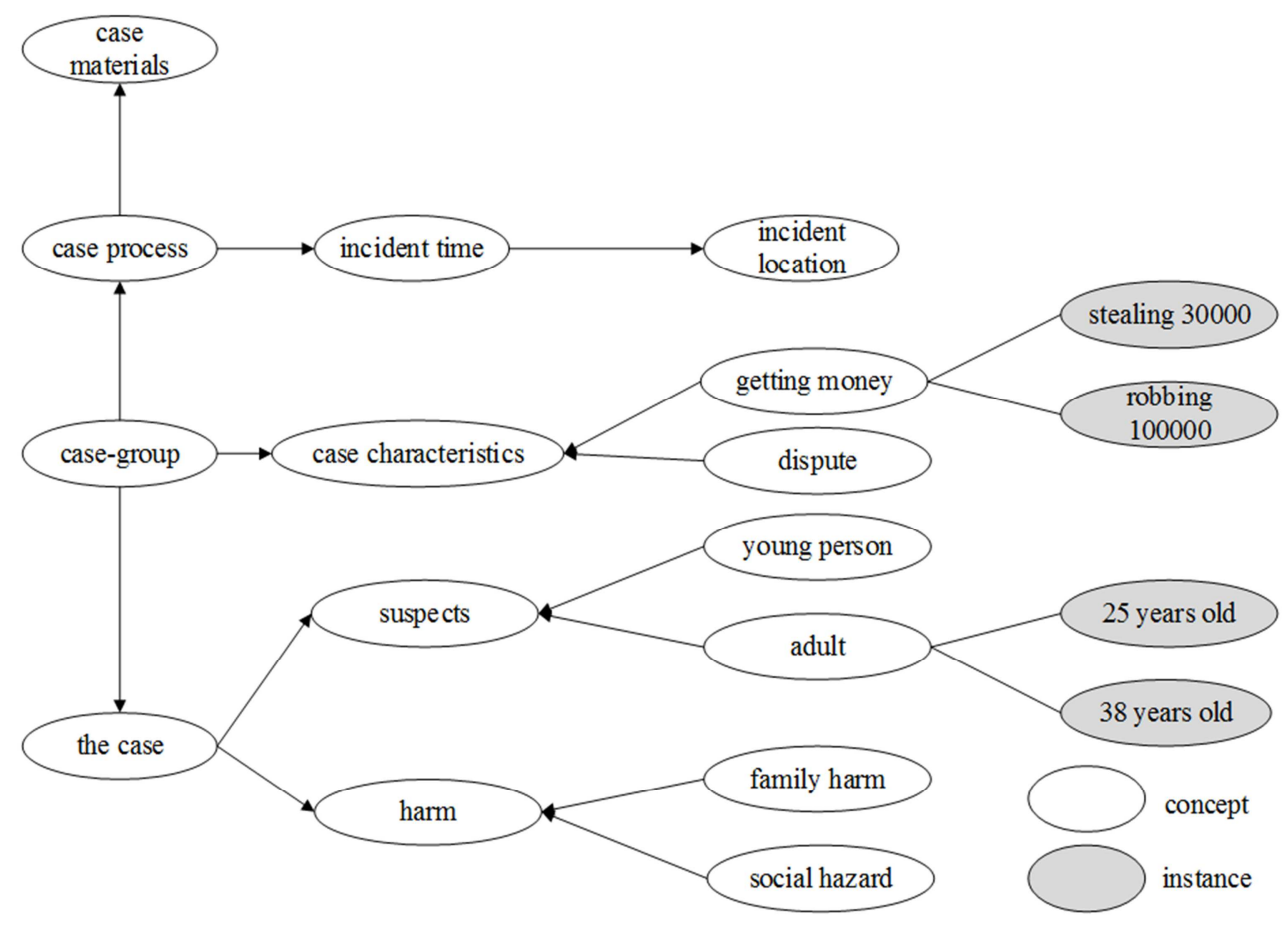

Figure 3. The simple ontology about the case.

\subsection{Stage Construction}

Stage construction is the core problem in the process of ontology construction. The process of case investigation is carried out by stages. Each stage is a sub-model which can be constructed by defining the scope of each stage, identifying the concepts, attributes, relations and formalizing expressions.

Definition for the scope of each stage: The research object of each stage involved in the investigation knowledge determines the scope of each stage and its scope covers the concerned details, since the stage construction is an important part of building the investigation ontology.

The identification of concepts, attributes and relationships: there are major concepts, attributes and relationships at all stages of the investigation process. Concept is an abstract summary of the essential characteristics of things, which has connotation and extension and changes with the development of things. An attribute is a description of an entity, which owns properties such as data type, domain, default value and so on. There are many concepts and relationships among concepts for an ontology.

Formalization: In the process of investigation, formal expression requires a meta-model to describe the acquired knowledge. It contains defining the main concepts, attributes, relationships and describing the details of the concepts, most of which need to be described by attributes and relationships to add the necessary constraint condition.

Upon these introductions, the first stage of investigation is that the ontology of receiving cases will be constructed. There are a series of steps in the stage of receiving cases, including the jurisdiction of the case, whether to accept the case, the initial review before case-filing, etc. The key concepts in the stage of receiving cases include "Filing standard", "Initial review" and so on. In order to describe the process of receiving stage in detail, the key attributes are the description attributes of these concepts, including the basic information such as "time", "manager", etc. The key concept is to describe the relationship between the concept "Filing" and "Precautions" in order to comprehensively describe the process of the receiving stage. There are also precautions in the case, such as the relationship "inclusive-in-filing". For the receiving stage of a case, conceptual details need the key relationship "related to on-filing" to describe the concept of filing and add the necessary constraints. For example, the relationship "includes-in-filing" is used to describe the concept "Filing".

Filing $\exists$ includes - in - filing Pr ecautions, "Filing" includes "Precautions" in the receiving stage. And "Precautions" is an attention-needed part in the filing stage, but it does not only contain "Precautions". Figure 4 is a fragment of the receiving case stage, which describes the 
top-level conceptual structure of the case-receiving stage. The details are defined as follows.

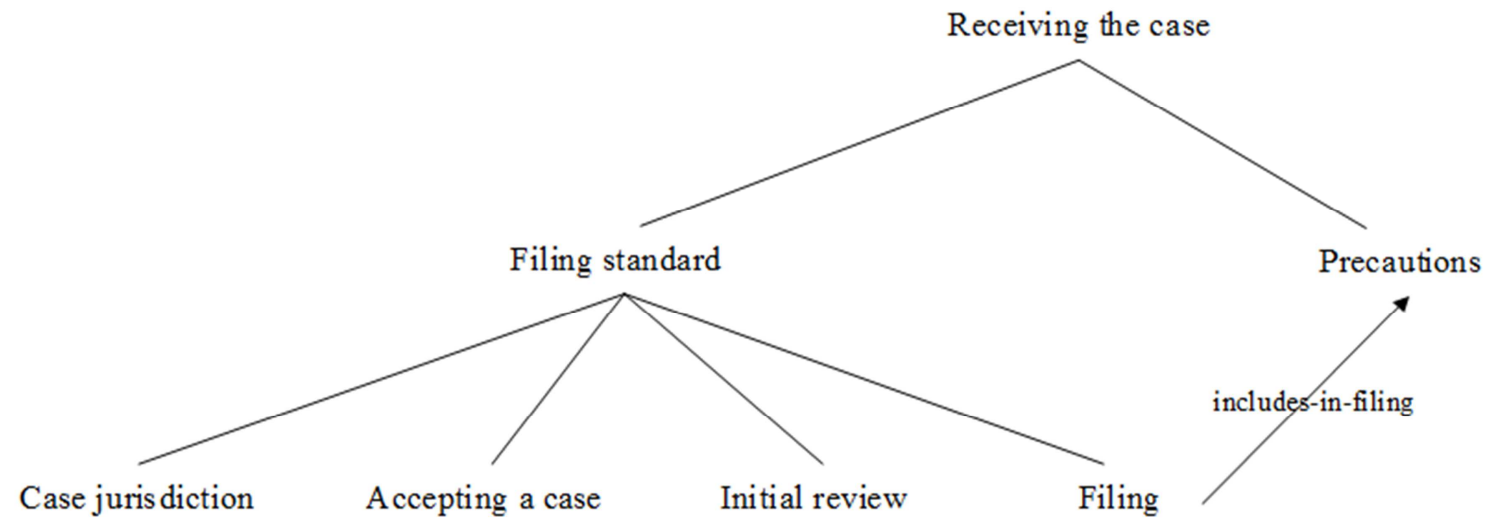

Figure 4. A fragment of receiving the case. (An arrow line between two concepts represents the key relationship).

Definition 1 (Case reception) Case reception is the first stage in the process of investigation, which is recorded as $R_{e c}$.

$$
R_{e c}=\left(P_{r e}, R_{f . s e t}, S_{t . s e t}\right)
$$

$\mathrm{P}_{\mathrm{re}}=$ Precautions,

$\mathrm{R}_{\mathrm{f} . \text { set }}=$ \{includes-in-filing, related-to-on- filing $\}$,

$\mathrm{S}_{\mathrm{t} . \mathrm{set}}=\{$ Case-jurisdiction, Accepting a case, Initial-review, Filing\}

In the stage of crime scene investigation, it covers different aspects of scene investigation, including investigation preparation, scene protection, scene search and so on. The key concepts of scene investigation are the related concepts of survey process to model the process of scene investigation, including "Survey preparation", "Field protection", "On-site search", "Fixed evidence", and "Transportation and preservation". The key attributes of scene investigation are the description attributes of related concepts to describe the scene survey process in detail, it including the basic attributes of the equipment such as "number", "name", "type" and so on. In order to describe the scene investigation fully, its key relationship is to describe the relationship between the concept "on-site search" and other steps, such as the relationship "has-result-data". At the same time, key attributes and relationships are used to describe the relationship between the concept "On-site search" and other concepts and add the necessary constraints. For example, the relationship "has-result-data" can be used to describe the concept "on-site search":

On - site search $\forall$ has - result - dataFixed evidence Formalization is identified by defining the scope and identifying concepts, attributes and relationships of this stage. The above steps are used to construct the second stage of the investigation, namely the stage of scene investigation. Figure 5 is a segment of the scene investigation, describing the top-level conceptual structure of the scene investigation. The details are defined as follows.

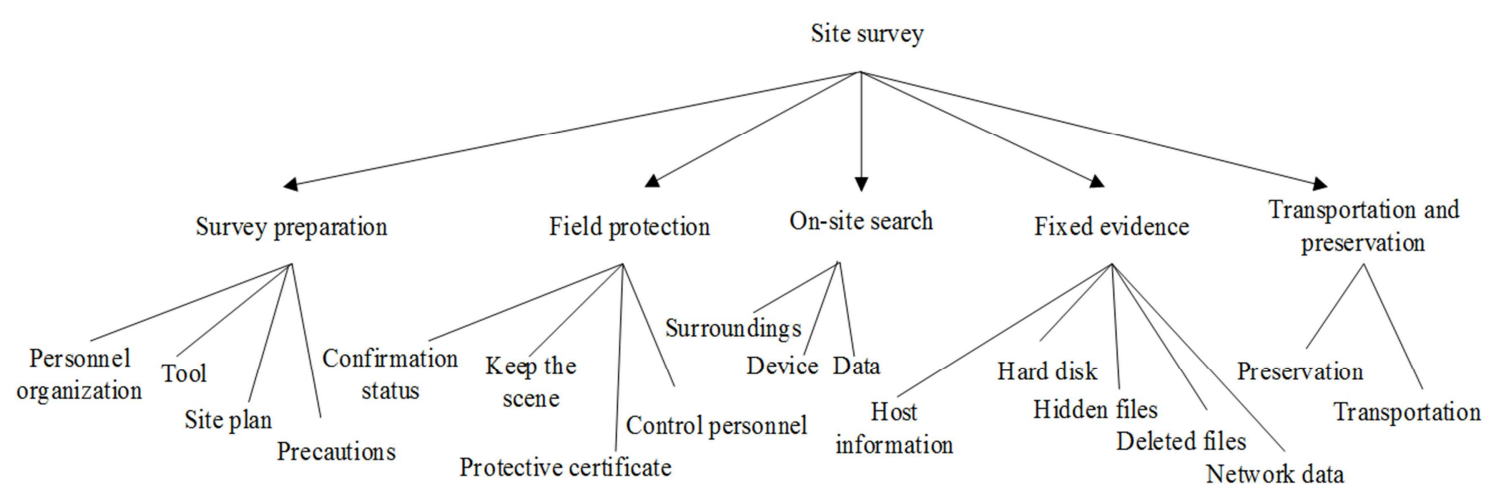

Figure 5. A fragment of the site survey.

Definition 2 (Site survey) Site survey is an important and second stage in the investigation process. It is significant for investigators to understand the case, to determine the direction of investigation and to make survey plans. It is often regarded as the breakthrough for the case investigation. Recorded as $S_{i t}$,

$$
S_{i t}=\left(S_{u r}, F_{i e}, O_{n s}, R_{s . s e t}, F_{i x}, T_{r a}\right)
$$

$S_{u r}=\{$ Personnel organization, Tool, Site plan, Precautions\},

$F_{i e}=\{$ Confirmation status, Keep the scene, Protective certificate, Control personnel $\}$,

$O_{n s}=\{$ Surroundings, Device, Data $\}, R_{\text {s.set }}=\{$ has-result-data, performs-survey $\}$,

$F_{i x}=\{$ Host information, Hard disk, Hidden files, Deleted files, Network data\},

$T_{r a}=\{$ Preservation, Transportation $\}$ 
The third stage of the investigation process is the stage of clue analysis and it is constructed through above steps. The key concepts in the stage of clue analysis contain "Types of clue", "Thinking of analysis", "Expansion methods" and "Comprehensive analysis". Key attributes are descriptions of concepts, such as the information attributes of the host clues such as "time", "message", etc. The key relationships are used to describe the connections between concepts, for example, the relationship is "uses". Through defining the scope and recognizing related concepts, attributes, relationships of this stage, formalization describes related concepts of the clue analysis with key attributes or relationships and adds corresponding constraints. For example, the relationship "uses" describes the concept "Network clues" as follows:

Network clues $\exists$ uses Tools, that is network clues can use tools.

After receiving the case and investigating the scene in the early stage, investigation enters into the stage of clue analysis and it covers all the clues that need to be analyzed. Figure 6 is a fragment of the clue analysis, describing the top-level conceptual structure of clue analysis. The details are defined as follows:

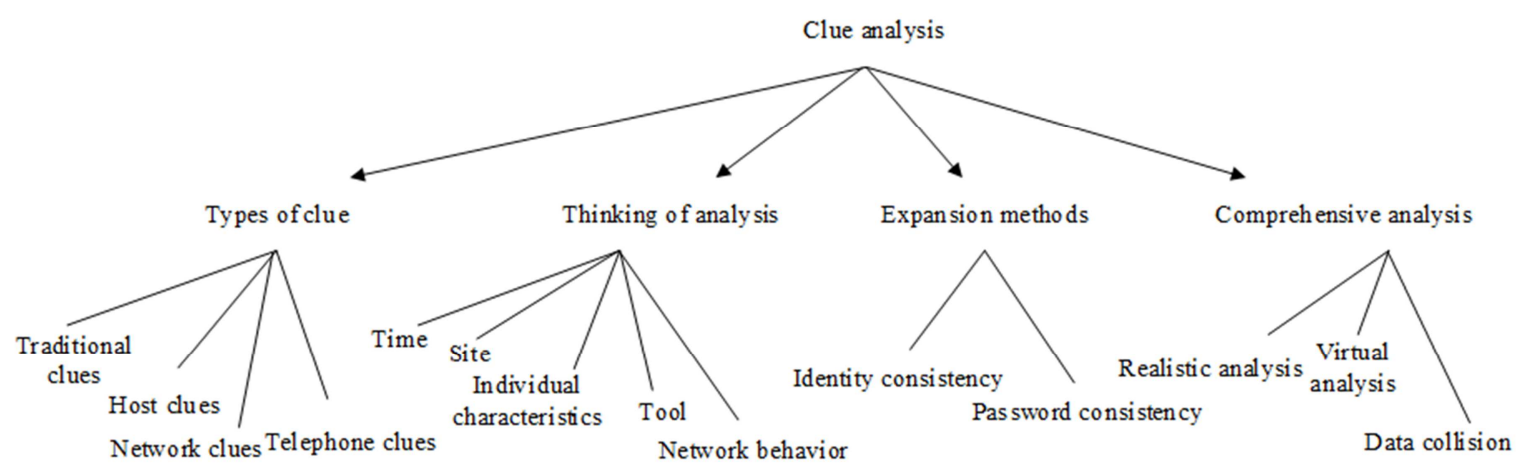

Figure 6. A fragment of the clue analysis.

Definition 3 (Clue analysis) Clue analysis runs through the whole process of case investigation. Whether clue analysis is successful determines the success or failure of the investigation process. The end of clue analysis means that the leading work of the investigation process is over. Recorded as $C_{l u}$

$$
C_{l u}=\left(T_{y p}, T_{h i}, R_{c . s e t}, E_{x p}, C_{o m}\right)
$$

$T_{y p}=\{$ Traditional clues, Host clues, Network clues, Telephone clues\},

$T_{h i}=\{$ Time, Site, Individual characteristics, Tool, Network behavior $\}, R_{\text {c.set }}=\{$ uses $\}$,

$E_{x p}=\{$ Identity consistency, Password consistency $\}$,

$C_{o m}=\{$ Realistic analysis, Virtual analysis, Data collision $\}$

After the previous stages of receiving cases, scene investigation and clue analysis, investigation enters into the last stage of identifying suspects. At this stage, the scope of this stage is defined firstly, which covered different aspects of identifying suspects, including identity clues, behavioral characteristics, etc. Key concepts contain "Identity clues", "Behavioral characteristics", "Psychological characteristics" and so on. Key relationships are used to describe the connections between concepts such as the "has-origin-data". In the stage of identifying suspects, the formal representation focuses on conceptual details, using key attributes or relationships to describe related concepts and adding the necessary constraints. For example, the relationship "has-origin-data" is used to describe the concept "Behavioral characteristics" as follows.

Behavioral characteristics $\forall$ has-origin-data custom The original data of "Behavioral characteristics" contains "custom". Figure 7 is a fragment for the identification of suspects, describing the top-level conceptual structure of identifying suspects. The details are defined as follows.

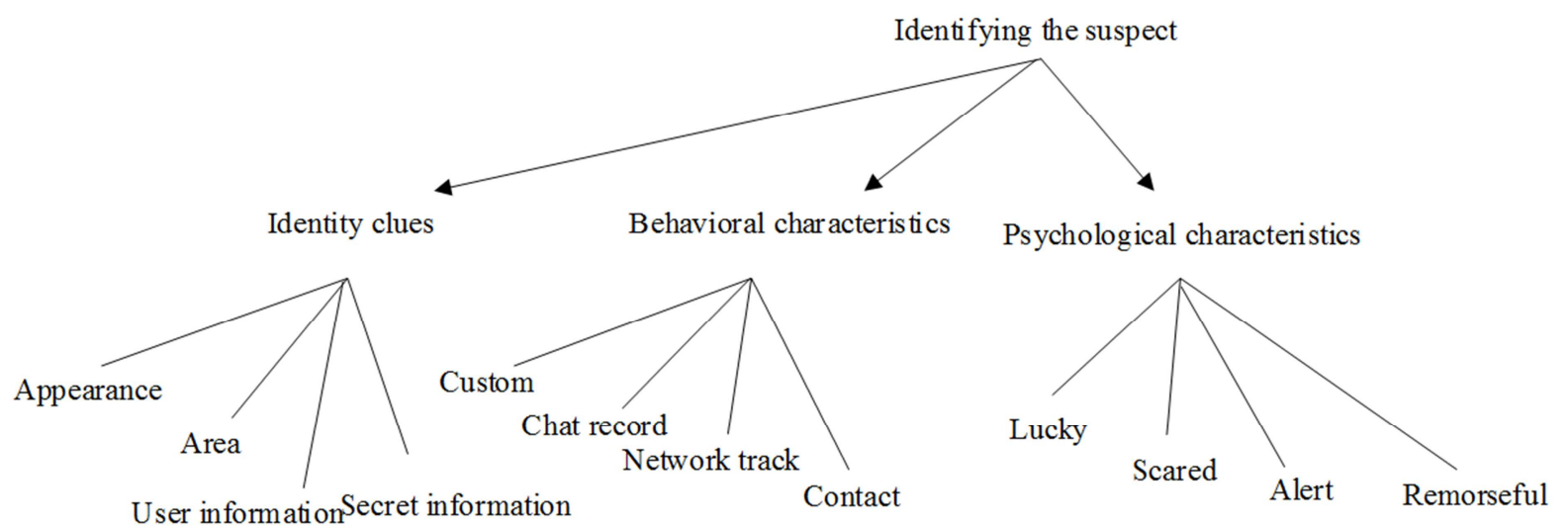

Figure 7. A fragment of identifying the suspect. 
Definition 4 (Identifying the suspect) Identifying suspects means the end of the investigation process. Recorded as $I_{d s}$

$$
I_{d s}=\left(I_{d c}, B_{e h}, R_{i . s e t}, P_{s y}\right)
$$

$I_{d c}=\{$ Appearance, Area, User information, Secret information\},

$B_{\text {eh }}=\{$ Custom, Chat record, Network track, Contact $\}$, $R_{\text {i.set }}=\{$ has-origin-data $\}$,

$P_{s y}=\{$ Lucky, Scared, Alert, Remorseful $\}$

\subsection{The Construction of Inter-stage Relationship}

The above-mentioned case reception, scene investigation, clue analysis and suspect identification have been

$$
\text { has - case data }=\left\{\left(i_{i}, i_{j}\right) \mid \exists C_{p}, C_{q}, C_{p} \in R_{e c} \cdot S_{t . s e t} \wedge C_{p}\left(i_{i}\right) \wedge C_{q} \in S_{i t} \cdot S_{u r} \wedge C_{q}\left(i_{j}\right)\right\}
$$

Definition 6 (has-partial clue) Has-partial clue is a kind of relationship between stages, which exists between the fixed evidence in the scene survey stage and corresponding clue

$$
\text { has }- \text { partialclue }=\left\{\left(i_{i}, i_{j}\right) \mid \exists C_{p}, C_{q}, C_{p} \in S_{i t} \cdot F_{i x} \wedge C_{p}\left(i_{i}\right) \wedge C_{q} \in C_{l u} \cdot T_{y p} \wedge C_{q}\left(i_{j}\right)\right\}
$$

Definition 7 (has-legal evidence) Has-legal evidence is a kind of relationship between stages. It exists between the traditional concept of clue in the clue analysis stage and the

$$
\text { has - legal evidence }=\left\{\left(i_{i}, i_{j}\right) \mid \exists C_{p}, C_{q}, C_{p}\right.
$$

The definitions of 5,6 , and 7 indicate that the same relationship can exist between multiple concepts. They describe the connection of multiple concepts among the four stages and connect the four stages effectively.

\subsection{The Evaluation and Evolution for an Ontology}

Through the steps described above, an ontology of investigation knowledge can be constructed, which is defined as follows.

Definition 8 (Investigation Ontology) The case investigation ontology describes the implementation of the investigation. It is a four-tuple and is denoted as $\mathrm{Oto}_{i}$.

$$
\text { Oto }_{i}=\left(\mathrm{Sec}_{\text {oto.set }}, C_{\mathrm{sec} . s e t}, R_{\mathrm{sec} . s e t}, X_{\mathrm{sec} . s e t}\right)
$$

$S e c_{\text {oto.set }}=\left\{R_{e c}, S_{i t}, C_{l u}, I_{d s}\right\}$ is a stage set.

$C_{\text {sec.set }}=\left\{P_{r e}, S_{t . s e t}, S_{u r}, F_{i e}, O_{n s}, F_{i x}, T_{r a}, T_{y p}, T_{h i}, E_{x p}, C_{o m}, I_{d c}\right.$, $\left.B_{e h}, P_{s y}\right\}$ is the content set.

$R_{\text {sec.set }}=\{$ has-case data, has-partial clue, has-legal evidence $\}$ is a set of relationships between stages.

$X_{\text {sec.set }}=\{O n$-site search $\forall$ has-result-data Fixed evidence,

Network clues $\exists$ uses Tools,

Behavioral characteristics $\forall$ has-origin-data custom, ...\} is a constraint set for inter-stage relationships.

The method of evaluating any ontology is indispensable in the process of constructing ontology. There are different types of evaluation methods for constructing ontology [35]. But the method of ontology evaluation for case investigation should be evaluated according to whether the investigation constructed respectively and realized the graph ontology. In order to construct the ontology of case investigation knowledge, these four ontologies need to be connected effectively. This paper established a relationships between stages for the progress of the investigation. Therefore, the relationships between stages as follows.

Definition 5 (has-case data) The relationship of "has-case data" is a relationship between stages. It exists between the concept of case filing in the case-receiving stage and the concept of survey preparation in the scene investigation stage. And it is used to describe the initial case and is recorded as has-case data. That is

analysis stage. It is used to describe the results of the scene survey, which is recorded as has-partial clue. That is

concept of user information in the suspect determination stage. The result of analyzing clues is recorded as has-legal evidence, that is

$$
\left.\in C_{l u} \cdot T_{y p} \wedge C_{p}\left(i_{i}\right) \wedge C_{q} \in I_{d s} \cdot I_{d c} \wedge C_{q}\left(i_{j}\right)\right\}
$$

efficiency of cases is improved in the actual practice. And this is also the goal that building investigation ontology needs to be achieved. It needs to integrate investigation ideas and case characteristics so that investigators can investigate cases more efficiently.

The ontology's evolution of case investigation needs to enrich experts' knowledge constantly to describe it better through the ontology and to improve it further. At the same time, the modeling process needs to be promoted continuously with the development of investigation, receiving different opinions to combine with actual practice and serving for actual practice.

\section{Semantic Reasoning Based on the Case Ontology}

The semantics are defined based on the ontology language and have the reason function. This paper will adopt protégé to construct the ontology of case and carry out reasoning of cases. And this paper takes the theft case as an instance to carry out experiments.

\subsection{The Ontology Model of Theft Case}

The theft case generally refers to the criminal act involved in stealing public or private property for the purpose of illegal possession. It needs to collect common occurrence time, the place, victims' characteristics, criminal behavior and other terms of theft cases. And then the relationship 
between object attributes and classes is defined to construct the ontology model of the theft case.

The classes, instances and relationships between the theft model constitute a complex network structure of the theft ontology. For example, it contains the time, the place, criminal behavior, etc. They are displayed as shown in Figure 8.

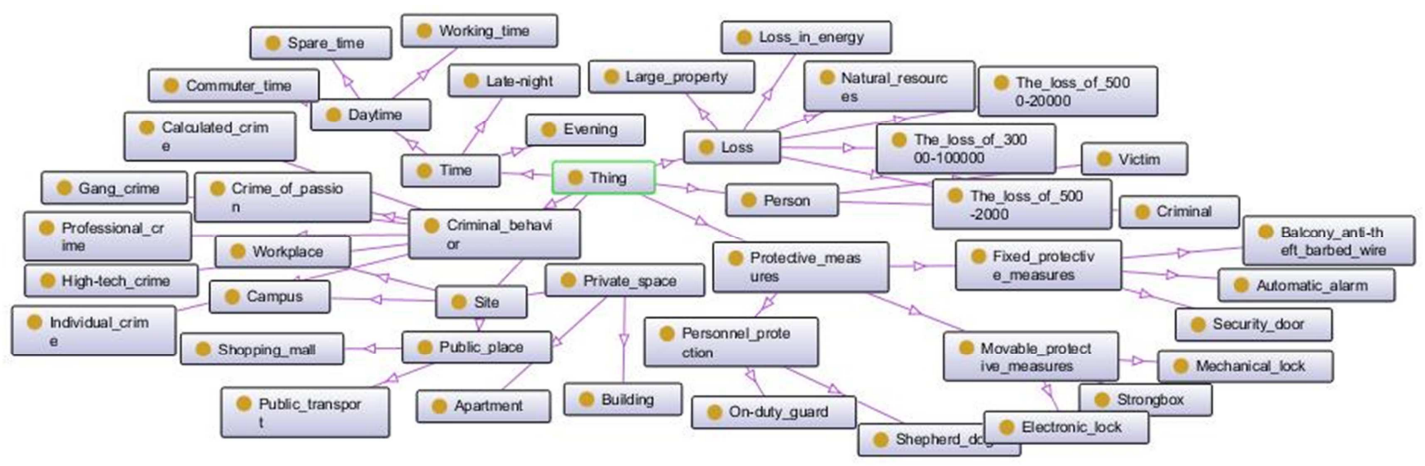

Figure 8. The network structure of theft ontology model.

\subsection{The Ontological Experiment of Semantic Reasoning for the Theft Case}

The criminal tools used in the theft case are taken as an example in the reasoning experiment of ontology semantics. It is summarized through examples of various theft cases, including electronic technology tools, electronic jammers, memory electronic card readers, violent physical tools, crowbar, technical physical tools, long scissors, etc. Criminals commit crime to steal property or other items through these criminal tools. Firstly, it needs to construct the class of criminal-tool and the class of items involved in the crime process and they are shown in Figure 9.

There are attributes of electronic stealing, violent theft and technical theft among the object properties. Electronic confrontation destruction is the sub-attribute of electronic stealing. Destruction is the sub-attribute of violent theft. Stealing is the sub-attribute of technical theft. And the object properties are constructed separately, as shown in Figure 10.

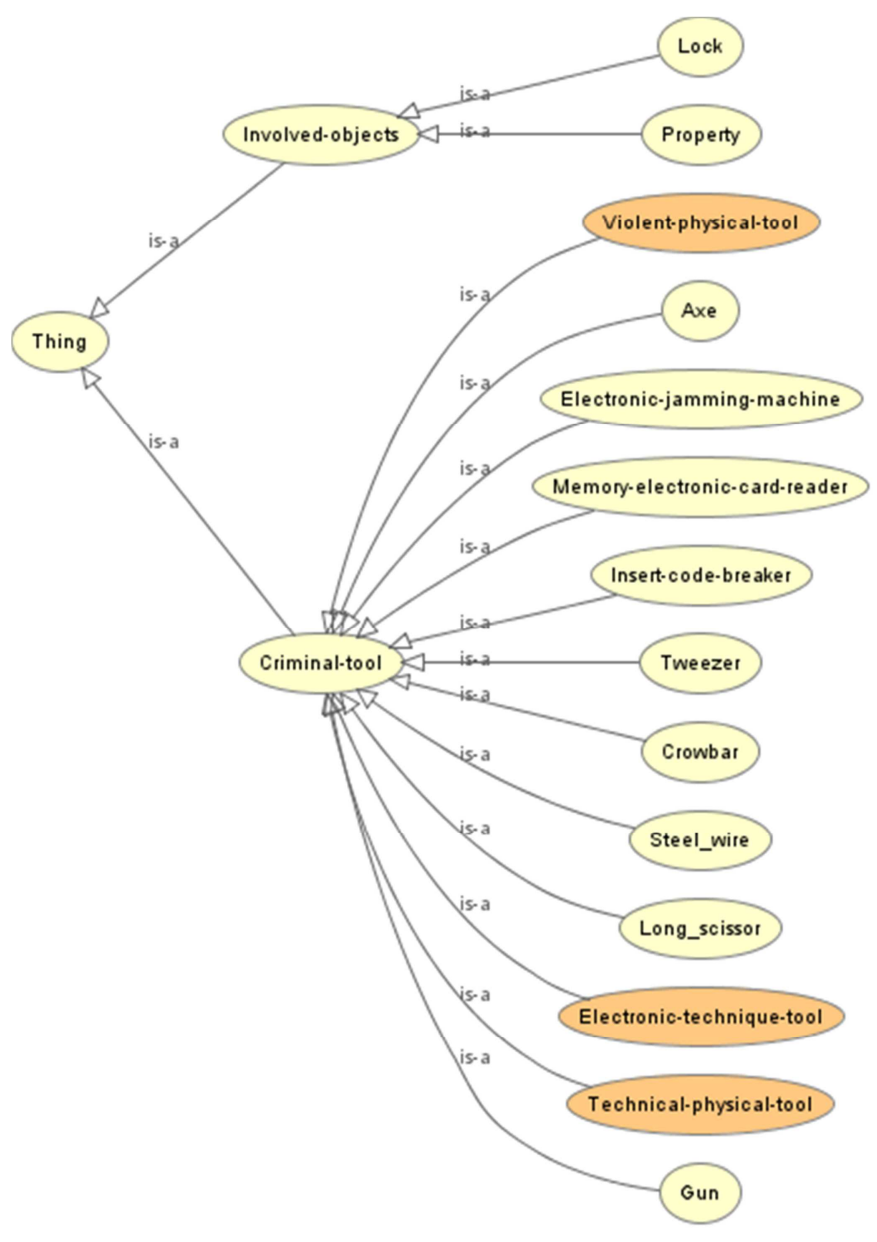

Figure 9. The class construction of criminal-tool and involved-objects. 


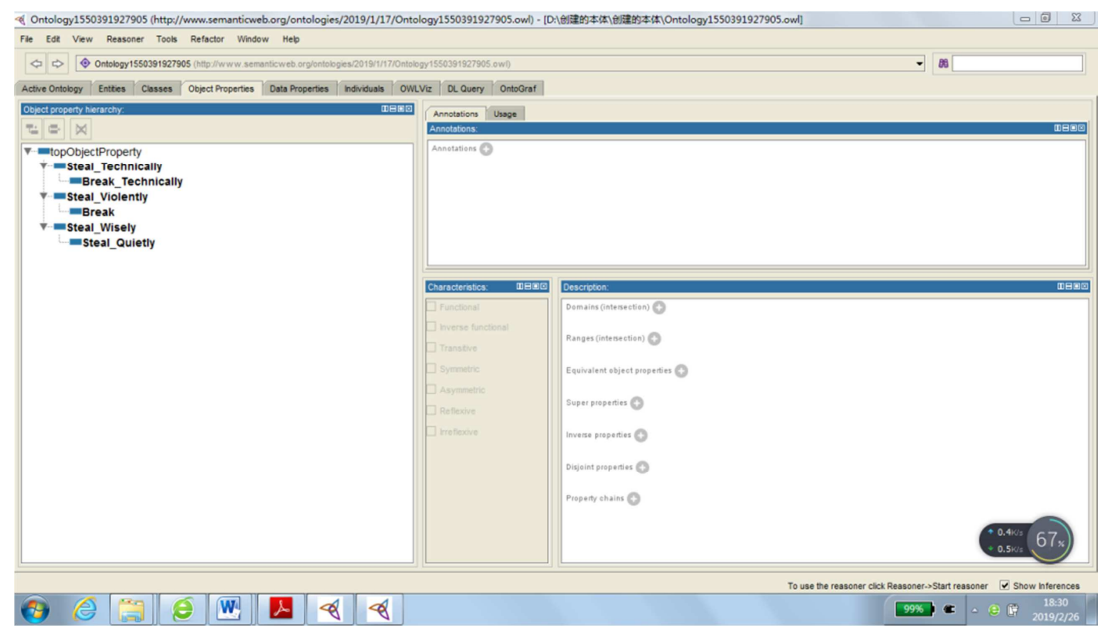

Figure 10. The constructed object properties.

Meanwhile, electronic technology tools can be interpreted as criminal tools, which steal relevant items technically. And that is the equivalent class of electronic technology tools, as shown in Figure 11. For example, the parent class of the axe breaks the lock by force.

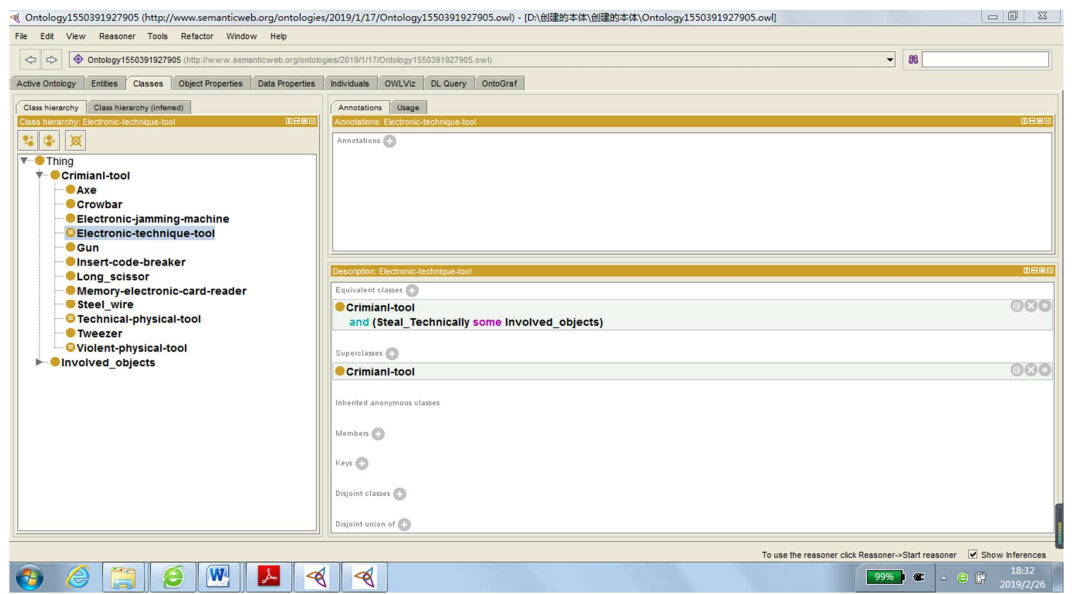

Figure 11. The equivalent classes of electronic-technique-tool.

So there are association rules about using criminal tools to commit theft. The violent physical tools use violent means to break the lock to carry out the theft. The technical physical tools steal goods by use of the tricky way. The electronic tools use electronic countermeasures to steal the lock. The reasoning is implemented, and the result is shown in Figure 12.

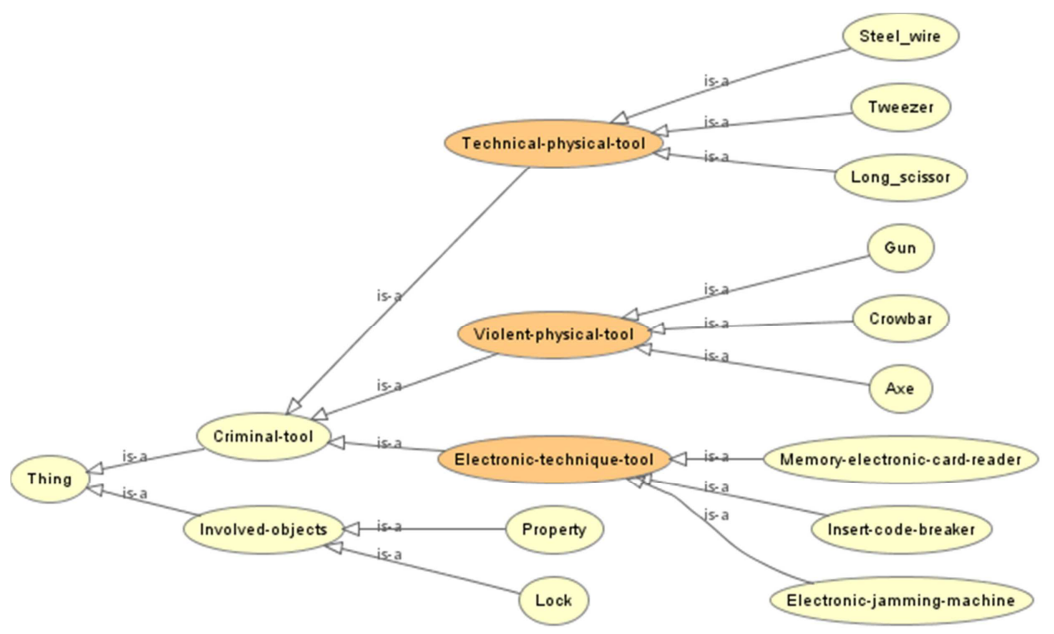

Figure 12. The class diagram after reasoning. 
As we can see from Figure 12 after realizing the reasoning, the result shows that the electronic jammer, the memory electronic card reader and the plug-in code lock decoder belong to electronic technology tools. The axe, the gun and the crowbar belong to violent physical tools. Tweezers, long scissors and steel wire belong to technical physical tools. The ontological construction of the theft case is not only to establish a knowledge system of cases, and what's more, it can deepen understanding of the internal stages or the links between stages and further identify the key knowledge and share it.

\section{Conclusions}

This paper organizes and integrates the knowledge of case clues based on the theory of ontology. It establishes the organization and the relationship of case process by studying the research achievements in other fields. In particular, the case knowledge ontology model is constructed through building the sub-ontology between stages. It is a tentative attempt about knowledge modeling in the cases based on ontology.

However, the ontology construction and the establishment of conceptual relations in the field need further discussion since there is unified standard for this aspect at present. In the background of big data, nowadays, there is a trend of carrying out knowledge integration and modeling in the field of public security. This paper is just provide a preliminary thought. We hope that more experts in this field communicate with each other and exchange ideas to make further contributions about serving the public security.

\section{Acknowledgements}

This work was supported by the JKF program of People's Public Security University of China (2019JKF334), and the National Key Research and Development Plan (2016YFC0801003).

\section{References}

[1] Xianjiao Zeng, Guangquan Xu, Xi Zheng, Yang Xiang, and Wanlei Zhou, E-AUA: An Efficient Anonymous User Authentication Protocol for Mobile IoT, IEEE Internet of Things Journal 6 (2): 1506-1519, April 2019. DOI: 10.1109/JIOT.2018.2847447.

[2] Guangquan Xu, Yao Zhang, Arun K Sangaiah, Xiaohong Li, Aniello Castiglione, $\mathrm{Xi}$ Zheng, CSP-E^2: An abuse-free Contract Signing Protocol with low-storage TTP for energy-efficient electronic transactions ecosystems, Information Sciences 476 (2019) 505-515, DOI: 10.1016/j.ins.2018.05.022.

[3] Guangquan Xu, Jia Liu, Yanrong Lu, Xianjiao Zeng, Yao Zhang, Xiaoming Li, A novel efficient MAKA protocol with desynchronization for anonymous roaming service in Global Mobility Networks, Journal of Network \& Computer Applications 107 (2018) 83-92.
[4] Zhenhu Ning, Guangquan Xu, Naixue Xiong, Yongli Yang, Changxiang Shen, Emmanouil Panaousis, Hao Wang, Kaitai Liang. TAW: Cost-Effective Threshold Authentication with weights for Internet of Things. IEEE Access 7: 30112-30125 (2019).

[5] Abdulaziz Alzubaidi, Swarup Roy, Jugal Kalita. A data reduction scheme for active authentication of legitimate smartphone owner using informative apps ranking, Digital Communications and Networks, 9 (2018) 1-9.

[6] Latif Ullah Khan. Visible light communication: Applications, architecture, standardization and research challenges, Digital Communications and Networks, 3 (2017) 78-88.

[7] Matthew L. Hale, Kerolos Lotfy, Rose F. Gamble, Charles Walter, Jessica Lin. Developing a platform to evaluate and assess the security of wearable devices, Digital Communications and Networks, 10 (2018) 1-13.

[8] Zhang Pengli, Chen Shiqu. Criminal investigation [M]. Beijing: Mass Press, 2010.5.

[9] Hao Hongkui. Discipline Status of Investigative Subject [J]. Public Security Education, 2002, (4).

[10] Bai L, Lao S Y, Smeaton A F. Video Semantic Content Analysis Based on Ontology [C] International Machine Vision and Image Processing Conference, Maynooth, Ireland, September 5-7, 2007.

[11] LI Zhi-yi, LI De-hui, ZHAO Peng-wu. Research on Automatic Extraction of Dontology Concept and Its Relation in E-commerce [J]. information science, 2018, 36 (7): 85-90.

[12] WEN Liang, LI Juan, LIU Zhiying, JIN Yaohong. A Method of Knowledge Representation and Ontology Modeling Based on Hierarchical Network of Concepts [J]. Journal of Chinese Information Processing, 2018, 32 (4): 66-73.

[13] WANG Mengxiang, RAO Qi, GU Cheng, WANG Houfeng. Metaphorical Knowledge Expression and Acquisition for Chinese Nouns $[\mathrm{J}]$. Journal of Chinese Information Processing, 2017, 31 (6): 01-09.

[14] Yang Xiaohui, Wan Rui, Zhang Haibin, Zeng Yifu, Liu Qiao. Semantical Symbol Mapping Embedding Learning Algorithm for Knowledge Graph [J]. Journal of Computer Research and Development, 2018, 55 (8): 1773-1784.

[15] JIANG Tianwen, QIN Bing, LIU Ting. Open Domain Knowledge Reasoning for Chinese Based on Representation Learning [J]. Journal of Chinese Information Processing, 2018, 32 (3): 34-41.

[16] GUAN Sai-Ping, JIN Xiao-Long, JIA Yan-Tao, WANG Yuan-Zhuo, CHENG Xue-Qi. Knowledge Graph Oriented Knowledge Inference Methods: A Survey [J]. Journal of Software, 2018, 29 (10): 01-29.

[17] Kaneiwa, K. and Mizoguchi, R., Distributed reasoning with ontologies and rules in order-sorted logic programming, Journal of Web Semantics 7 (3), pp. 252-270, 2009.

[18] XU Wei, LI Kai, WANG Yanlong. Ship cabin layout design ontology model oriented to semantic reasoning application [J]. Journal of Dalian University of Technology. 2018, 58 (5): 479-486. 
[19] Ma L., Yu H., Chen G., Cao L., Zhao Y., Research on Construction and SWRL Reasoning of Ontology of Maize Diseases. In: Li D., Chen Y. (eds) Computer and Computing Technologies in Agriculture VI. CCTA 2012. IFIP Advances in Information and Communication Technology, vol 393. Springer, Berlin, Heidelberg, 2013.

[20] Singh S., Kaur R., Analyzing and displaying of crime hotspots using fuzzy mapping method. International Journal of Computer Applications, 103 (1): 25-28, 2014.

[21] WANG Jiahai, CHEN Yu. Data-driven Job Shop production scheduling knowledge mining and optimization [J]. Computer Engineering and Applications, 2018, 54 (1): 264-270.

[22] Anna L Buczak and Christopher M Gifford. 2010. Fuzzy association rule mining for community crime pattern discovery. In ACM SIGKDD Workshop on Intelligence and Security Informatics. ACM, 2.

[23] Lau, R. Y., Y. Xia, and Y. Ye (2014). A probabilistic generative model for mining cybercriminal networks from online social media. IEEE computational intelligence magazine 9 (1), 31-43.

[24] CHEN Zhigang, LIU Zhikun, YANG Lujing. Modeling of a PO for Tactical Intention Recognition Based on PR-OWL [J]. Ship Electronic Engineering, 2015 (2): 86-89.

[25] Mittal, S., P. K. Das, V. Mulwad, A. Joshi, and T. Finin (2016). Cybertwitter: Using twitter to generate alerts for cybersecurity threats and vulnerabilities. In Advances in Social Networks Analysis and Mining (ASONAM), 2016 IEEE/ACM International Conference on, pp. 860-867. IEEE.

[26] TANG Zai-jiang, XUE Xiang-zhong, XUE Qing, HUO Biao. Operational Action Ontology Modeling and Semantic Reasoning Based on Ontology [J]. Computer Simulation, 2018, 35 (6): 1-6.

[27] BAI Liang, LAO Song-yang, LIU Hai-tao, BU Jiang, CHEN Jian-yun. Video Semantic Content Analysis Using Extensions to OWL [J]. JOURNAL OF NATIONAL UNIVERSITY OF DEFENSE TECHNOLOGY, 2010, 32 (2): 79-84.
[28] Jie Ma; Jing Zhang; Hong Lu; XiangyangXue; "Semantic Information Extraction of Video Based on Ontology and Inference," Semantic Computing, 2007. ICSC 2007. International Conference on, vol., no., pp. 721-726, 17-19 Sept. 2007.

[29] Heum Park, SunHo Cho, and Hyuk-Chul Kwon. Cyber Forensics Ontology for Cyber Criminal Investigation. LNICST 8, 160-165, 2009.

[30] Raj Kumar Vishwakarma, Ravi Shankar. Modeling Brain and Behavior of a Terrorist through Fuzzy logic and Ontology. Proceedings of the 2013 IEEE IEEM, 2-7, 2013.

[31] Banczyk, K.; Krawczyk, H.; "Ontology Oriented Threat Detection System (OOTDS)," Dependability of Computer Systems, 2009. DepCos-RELCOMEX '09. Fourth International Conference on, vol., no., pp. 144-151, June 30 2009-July 22009.

[32] Mirna El Ghosh, Hala Naja, Habib Abdulrab, Mohamad Khalil. Towards a Legal Rule-Based System Grounded on the Integration of Criminal Domain Ontology and Rules. 21th International Conference on Knowledge Based and Intelligent Information and Engineering Systems, 632-642, 2013.

[33] Horrocks, I., Kutz, O., Sattler, U., The even more irresistible SROIQ, In Proc. of the 10th Int. Conf. On Principles of Knowledge Representation and Reasoning, 2006, pp. 57-67, AAAI Press.

[34] Xu Z M, Cao X, Dong Y S, Su W P. Formal approach and automated tool for translating ER schemata into OWL ontologies. Proceedings of the 8th Pacific-Asia Conference on Advances in Knowledge Discovery and Data Mining (PAKDD 2004), Sydney, Australia, 2004. Springer, 464-476, 2004.

[35] Brank J, Grobelnik M., Mladenic D. A survey of ontology evaluation techniques. Proceedings of the 4th Conference on Data Mining and Data Warehouses (SiKDD 2005), Ljubljana, Slovenia, 2005: 166-169. 